\title{
Time resolved particle dynamics in granular convection
}

\author{
J.M. Pastor ${ }^{\mathrm{a}}$, D. Maza ${ }^{\mathrm{a}}$, I. Zuriguel ${ }^{\mathrm{a}}$, A. Garcimartín ${ }^{\mathrm{a}, *}$, J.-F. Boudet $^{\mathrm{b}}$ \\ ${ }^{a}$ Depto. de Física y Mat. Apl., Facultad de Ciencias, Universidad de Navarra, 31080 Pamplona, Spain \\ ${ }^{\mathrm{b}}$ CPMOH, Université de Bordeaux I, 33405 Talence Cedex, France \\ Received 31 May 2007; accepted 9 June 2007 \\ Available online 21 June 2007 \\ Communicated by R.P. Behringer
}

\begin{abstract}
We present an experimental study of the movement of individual particles in a layer of vertically shaken granular material. High-speed imaging allows us to investigate the motion of beads within one vibration period. This motion consists mainly of vertical jumps, and a global ordered drift. The analysis of the system movement as a whole reveals that the observed bifurcation in the flight time is not adequately described by the Inelastic Bouncing Ball Model. Near the bifurcation point, friction plays an important role, and the branches of the bifurcation do not diverge as the control parameter is increased. By fitting the grains trajectories near the wall it is possible to quantify the effective acceleration acting on them. A comparison of the mass centre flying time and the flying time determined for the grains near the wall exposes the underlying mechanism that causes the downward flow.
\end{abstract}

(C) 2007 Elsevier B.V. All rights reserved.

Keywords: Granular flow; Convection

\section{Introduction}

Granular convection is a patent example of how collective movement of grains can give rise to an ordered yet complex behavior. As soon as 1831, Faraday [1] reported a long range flow developed in a vertically shaken granular layer. This flow is called granular convection because of the likeness between it and the movement of a liquid layer heated from below. Although many works have dwelt on this topic, the origin of this convective movement, and in particular the role of the lateral walls or the boundaries, is not fully understood. In 1989, Evesque and Rajchenbach [2] published an article where they showed experimentally that the threshold for collective motions to appear corresponds to the acceleration of gravity $g$. This is why the acceleration of the external driving is often given in the form of an adimensional number $\Gamma=\frac{A \omega^{2}}{g}$, where $A$ is the amplitude and $\omega$ is the frequency of the forcing. They also described how a heap grows changing the shape of the

\footnotetext{
* Corresponding author. Tel.: +34 948 425600; fax: +34 948425649 .

E-mail address: angel@ fisica.unav.es (A. Garcimartín).
}

free surface of the medium, as a consequence of the grains circulating in a "convective" fashion.

Almost at the same time, Laroche et al. [3] reported both the importance of interstitial air for the deformation of the granular layer and the development of a compactation front that splits the layer into two zones, a solid one and a liquid one. The origin of convection, according to these authors, would be directly related to the air circulating among the grains. This effect determines the rising of material at the center of the layer and a flow of grains going down near the walls, which influence the material by increasing its porosity with respect to the central zone. Nevertheless, subsequent studies [4,5] have revealed that the walls can by themselves provide the driving force for convection, at least for a two-dimensional geometry. This point was finally demonstrated by the works of the Chicago group [6], who used NMR techniques to show that wall friction does affect the velocity profile of the particles. It should be noted that the shaking was conveyed in this case in the form of short pulses, or "taps", separated by rest periods much longer than the pulse itself. At the same time, enlightening ideas were put forward, and tested numerically, setting the framework in which to understand the collective behaviour of 
granular matter. Following an analogy with hydrodynamics, models were developed that qualitatively predicted the longrange ordering of a shaken granular media, even though simplifications sometimes made them unrealistic [7-10].

Above the convective threshold, a granular layer can also undergo a rich array of instabilities. In 1989 Douady et al. [11] showed that beyond a certain value of $\Gamma$ the flight of the grains experiences a period doubling bifurcation, in a way essentially equivalent to a solid body that is placed on a vibrating plate [12]. As a layer of granular material is strongly dissipative, it can be considered perfectly inelastic, and its behaviour as a whole can be modelled by an inelastic ball on a vibrating plate. This simple model, known as the Inelastic Bouncing Ball Model (IBBM) has been discussed by several authors [13-15] and successfully used to describe the temporal dynamics of a shallow granular layer (without convection) [16] as well as the dilatation of a thick granular layer [17]. Moreover, as the system is spatially extended, it can also undergo spatial instabilities associated with the breaking of translational symmetries between different zones of the layer, that can oscillate with different phases [16].

More recently, it has been shown how the convective velocity field depends on the adimensional acceleration and other parameters, such as frequency and the air pressure [18,19], and new theoretical models have been developed [20-22].

In this article we present an experimental study of the motion that a dense granular system develops when it is submitted to a sinusoidal vibration in the same direction as gravity. By detecting the time at which the layer collides with the shaking plate, flight times of the granular layer as a whole are measured, and the temporal bifurcations are described. At the same time, the movement of the particles near the container walls has been tracked with a high-speed recording system. By tracking the grains within an oscillation period, the friction effects caused by walls can be quantified and its influence on the global circulation is assessed.

\section{Experimental set-up}

Convection can be observed with almost any granular material, irrespective of shape, size or surface features. We have used glass beads with a diameter of $0.5 \pm 0.1 \mathrm{~mm}$, but we checked that sand gives the same qualitative results. The relative effect of cohesive forces (such as humidity or static charges) is important if the beads are much smaller than this. On the other hand, it is desirable to have as many grains as possible, and this particular size offers a good compromise. We put a big number of beads (typically of the order of $10^{4}$ ) inside a cylindrical box made of glass. The fact that both the beads and the box are of the same material reduces the amount of electrical charges created by friction. We also sprinkled the box with antistatic spray. The diameter of the box is $52 \mathrm{~mm}$ and it is high enough to avoid grains falling out when vibrated. This box is attached to a TiraVib 52100 magnetic shaker capable of delivering a sinusoidal acceleration of up to $15 \mathrm{~g}$ with a distortion smaller than $0.05 \mathrm{~g}$. The shaker is commanded by a Stanford Research DS345 function generator. The vibration is

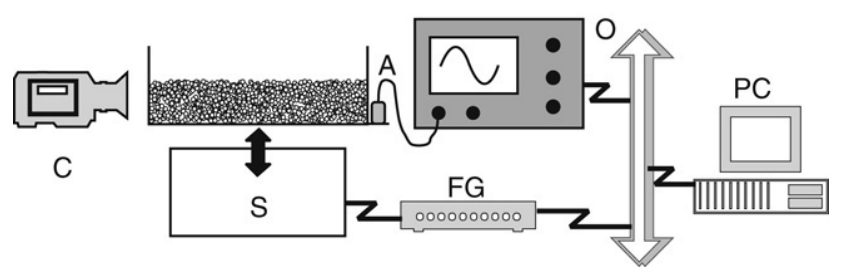

Fig. 1. Experimental set-up. A granular layer is vibrated by means of a shaker (S), which is in turn controlled by a function generator (FG). The acceleration is measured with an accelerometer (A). An oscilloscope (O) is used to monitor the instantaneous acceleration. The movement of the particles adjacent to the wall is recorded with a high-speed camera (C). The devices are controlled from a PC.

characterized with an accelerometer attached to the box that has a sensitivity of $100 \mathrm{mV} / \mathrm{g}$, whose signal is picked up by a Hewlett-Packard HP54510 digitizing oscilloscope. Both the oscilloscope and the function generator are connected to a PC. A sketch is provided in Fig. 1.

The frequency of the external vibration $f$ was kept constant at $f=110 \mathrm{~Hz}$ (so $T=0.00909$ ). We had previously found that the features of convection do not change qualitatively with frequency [18] provided that it is higher than $60 \mathrm{~Hz}$. The acceleration was therefore changed by regulating the vibration amplitude $A$. The size of the granular layer is given in terms of the dimensionless height $N=h / \phi$, where $h$ is the thickness of the layer and $\phi$ the particle diameter.

We used a high-speed camera (Motionscope Redlake, model 1105-0003) with a macro lens and a VCR to record the movement of the grains at 1000,2000 or 4000 frames per second. Under proper illumination, each glass sphere will reflect a bright spot that has been tracked with the following procedure. Once transferred to the computer, the movie was split into individual frames. A morphological image processing was performed on each frame to obtain the centroid of one bright spot in the first recorded frame. As the spheres move less than one diameter from one frame to the next, the position of the bright spot is easily identifiable in the subsequent frame. Repeating the procedure for all the recorded frames and by tracking several beads, a set of grain positions versus time was obtained from each movie. Note that only spheres adjacent to the walls are accessible with this method, and we can only measure the velocity in the plane of the wall. An alternative method that has also been used, yielding the same results, is to calculate the correlation function between consecutive frames. In this case, the averaged velocity of all the beads in the frame is obtained.

\section{Motion of the centre of mass}

Let us begin by describing the motion of the layer as a whole without considering the movement of the individual grains. Under this assumption, and considering the layer as a perfectly inelastic solid, its center of mass will begin to fly when its acceleration overcomes gravity. From then on, the material will perform a free flight, and will lose all its energy when it collides with the plate. If the acceleration of the container is at that moment smaller than $g$, as in Fig. 2(a), the layer gets stuck to the container base until it departs from the base again when the 


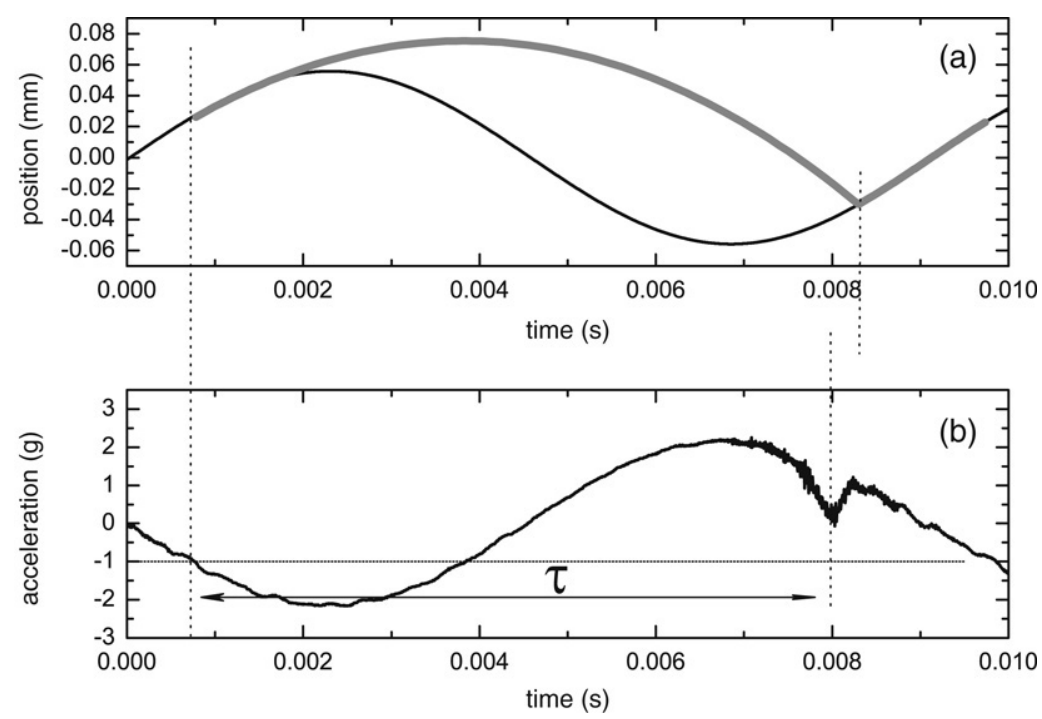

Fig. 2. Parabolic flight predicted by the Inelastic Bouncing Ball Model (a) and the acceleration measured by the oscilloscope for $\Gamma=2.37$ and $N=33$ (b). The collisions of the granular layer against the base of the container are evident in the signal from the accelerometer. The value of the acceleration equal to $-g$ is marked with a horizontal dotted line; the granular layer gets loose at this coordinate. Remarkably, the flight time measured in the experiment, $\tau$ suffers a phase delay with regard to the predicted by the IBBM. Take off and collision are marked with vertical dotted lines.

acceleration exceeds the gravity. The layer spends therefore a time $\tau$ in the flight and a time $T-\tau$ (where $T$ is the period of the container oscillation) stuck to the container base in each cycle.

The flight time $\tau$ grows with $\Gamma$ until it reaches the value of an oscillation period of the forcing $T$. If the granular layer is considered as a point mass, this happens for $\Gamma=\sqrt{1+\pi^{2}}$. At that point, the flight time undergoes a saddle-node bifurcation with the stable branch corresponding to a flight time $\tau=$ $T$ [12]. This lasts until $\Gamma=\sqrt{4+\pi^{2}}$, where a period doubling bifurcation takes place. Beyond that point, the particle can either perform a long flight (longer than $T$ ) or a short flight (shorter than $T$ ), depending on the container acceleration at the time of the collision. As $\Gamma$ increases, the long flight grows longer and the short flight shorter. Above $\Gamma=\sqrt{1+4 \pi^{2}}$ only the long flight survives, and when it reaches the value $2 T$ it bifurcates again.

The validity of this model to reproduce the interaction of the granular layer as a whole with the vibrating plate can be assessed by comparing its predictions to the experimental measurement of $\tau$ (see Fig. 2(b)). In order to do this, we have taken the value predicted by the IBBM for the phase at the beginning of the flight: $\phi_{i}=\arcsin (1 / \Gamma)$. There is no way to obtain this value from the acceleration signal, as the take off does not leave any trace on it. In principle this value is not prone to be affected by the friction between the grains and the container or the presence of interstitial gas, because at take off the relative velocity between the granular layer and the vibrating plate is zero. The collision time can be obtained experimentally from the measured acceleration, as in Fig. 2(b). The flight times obtained in this way, subtracting the take-off times from the collision times, are displayed in Fig. 3(a) along with the bifurcation diagram predicted by the IBBM. Clearly the model reproduces quite well the flight times of the centre of mass for $\Gamma \lesssim 2.7$, as has already been demonstrated [17].

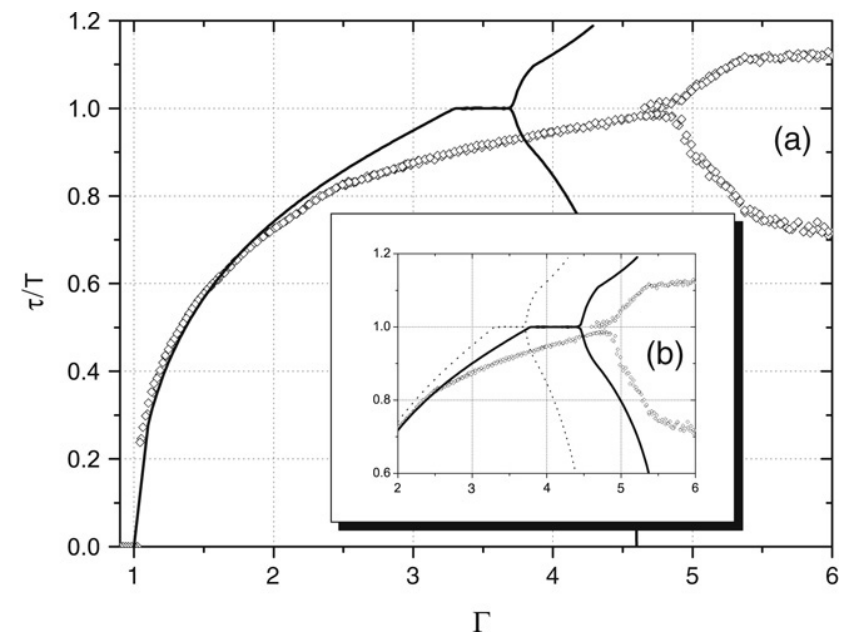

Fig. 3. Dimensionless flight time of the granular layer as measured from the acceleration signal (symbols). When $\tau$ reaches $T$ the flight time undergoes a period doubling: a long and a short flight are performed every two cycles. The line indicates the values predicted by the IBBM. The inset shows the same data but now the solid line is a numerical simulation including the air effects (as in the Kroll analysis). This analysis improves the fit up to $\Gamma \simeq 2.5$. The dotted line represents the IBBM.

Remarkably, just above this value, the model is not valid anymore. The measured flight times are shorter than those predicted, and the bifurcation point $(\Gamma=4.8 \pm 0.1)$ is $30 \%$ higher than the predicted one. Beyond that point, the branches grow but eventually they seem to saturate. Another remark is that the system bifurcates directly from a monotonically growing solution to a period two solution, without showing the saddle-node bifurcation predicted by the model. Even though the existence of such a bifurcation can not be completely rejected, we have been able to rule out that there is an extended region in which flight times hook to the oscillation period; if this region exists, it is much smaller than predicted. 
It is conceivable that one cause for this behaviour could be associated with the effects of the interstitial gas on the granular layer. The layer should then be considered as a porous medium whose porosity changes as it detaches from the base. A pioneering study of those dynamics has been done by Kroll [23] and refined by Gutman [24], who introduced air compressibility and a coupling with the porosity of the medium.

Let us introduce the hypothesis of Kroll (which is easier than Gutman's to perform) in the numerical simulation of our problem. Considering the inelastic ball as a porous piston interacting with the air in the cell (a similar analysis has been recently reported for the case of granular segregation [25]), the numerical analysis of the flight time suggests that air effects on the systems should be measurable but ought not to change the dynamics (see Fig. 3(b)), i.e. the saddle-node bifurcation is still present (its range of stability is even increased) and the branches diverge as the flight time approaches $2 T$.

In order to test experimentally the air influence on the flight time, we evacuated the container to $10^{-2}$ Torr and we collected data for the same range of $\Gamma$. Results are shown on Fig. 4(a). The agreement of measured data in vacuum with the IBBM is excellent up to $\Gamma \approx 2.3$, better than in the presence of air. Up to that value, flight times are a bit shorter if there is interstitial air. Nevertheless, above $\Gamma \approx 2.3$ the measured flight times do not fit to the IBBM even in vacuum. The bifurcation point is noticeably changed, even though it is still beyond the place predicted by the IBBM; the saddle-node bifurcation is also not observed. Beyond the bifurcation, the branches behave similarly in both cases (in vacuum and in air); a remarkable feature in vacuum is a region where flight times are bivaluated $(4.7<\Gamma<5)$. It is interesting to note the similarity of the branches between them and the likeness to the branch before the bifurcation point; this will be analysed elsewhere.

From these measurements, it is evident (Fig. 4) that the effect of the air on the flight time is equal to the expected one only for accelerations $\Gamma \simeq 2.5$. Beyond this value, the measured flight times are noticeable smaller than predicted for an inelastic particle including a viscous drag. Remarkably, this happens both in vacuum and in the presence of air: the flight times are almost equal in the two cases up to the bifurcation point. This fact suggests that the cause for this disagreement must be the same in both cases, and therefore different from the viscous effects due to the interstitial gas.

The key could be the interaction of the granular medium with the lateral wall of the container. Thus, the wall would exert an effective force on the inelastic ball different than gravity that would affect not only the initial phase of the flight [5] (its effects on the initial velocity being negligible) but the acceleration during the entire flight as well, resulting in an effective force applied in to the grains during the flight bigger than gravity in average. We estimate the magnitude of this force by comparing the measured flight times with those predicted by the IBBM. Let us introduce an effective control parameter such as $\Gamma_{\text {eff }}=$ $\frac{A \omega^{2}}{g_{\text {eff }}}$ which depends on an effective acceleration $g_{\text {eff. }}$. Form the measured flight time, we can consider that the system as a whole is moving under the action of an effective acceleration whose value is $10.6 \mathrm{~m} / \mathrm{s}^{2}$ if the flight time predicted for an

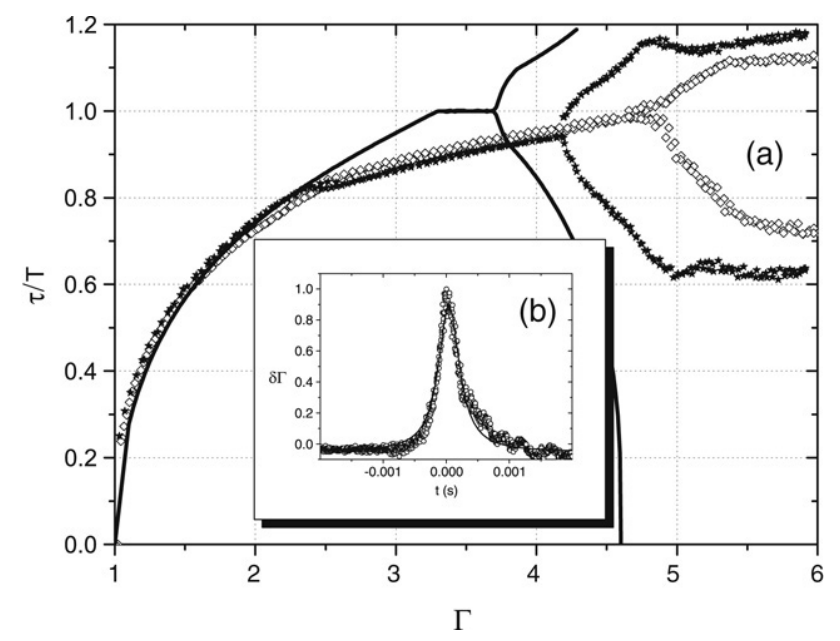

Fig. 4. Dimensionless flight time of the granular layer in an evacuated container (filled symbols). The agreement with the IBBM (solid line) is better than in air, but only for $\Gamma<2.3$. Above this value and up to the bifurcation, the dynamics of the system does not conform to the IBBM and coincides with the behaviour in the presence of air (open symbols). Inset: the collision retrieved from the measured acceleration ( (for $\Gamma=3$ ). The collision between the layer and the vibrating plate takes place during a finite time that can be measured from these data. The line is a lorentzian fit that is intended as a guide for the eye.

inelastic body is to be recovered. Certainly this is just a crude approach, but it highlights the fact that during the flight another forces - apart from gravity - are applied to the medium. This will be described in detail in the next section, where the movement of individual grains is dealt with.

But the existence of a net force larger than $g$ acting on the system is not enough to reproduce the flight time measured for $\Gamma>2.5$ up to the bifurcation point. A feature that is lacking in the model is the finite duration of the collision between the inelastic body and the plate. The extent of this time is a considerable portion of the oscillation period $T$, as can be appreciated in Fig. 4(b). This implies that the velocity of the centre of mass at take off is not necessarily well defined. If the collision lasts for some time, it is reasonable to think that there is a delay that leads to a decrease in the initial velocity of the center of mass, and therefore to shorter flight times. This phenomenon is also coupled with the propagation of a shock wave front [26] and it has significant consequences for $\Gamma>3$, when the duration of the collision becomes similar to the time that the granular layer spends stuck to the vibrating plate. For flight times shorter than 80 per cent of the period a finite collision time should not affect significatively the flight time, as we shall describe in the next section.

\section{The motion of individual grains}

Till here we have described the motion of the granular layer as a whole. But there is motion in the frame of reference of the layer: the convective flow. Let us now study the movement of individual particles. The convective motion - it has already been described $[18,6]$ - is much slower than the vibration, so it could somehow be expected that the motion of individual beads is a combination of flights similar to those of the IBBM coupled with a slow drift. 


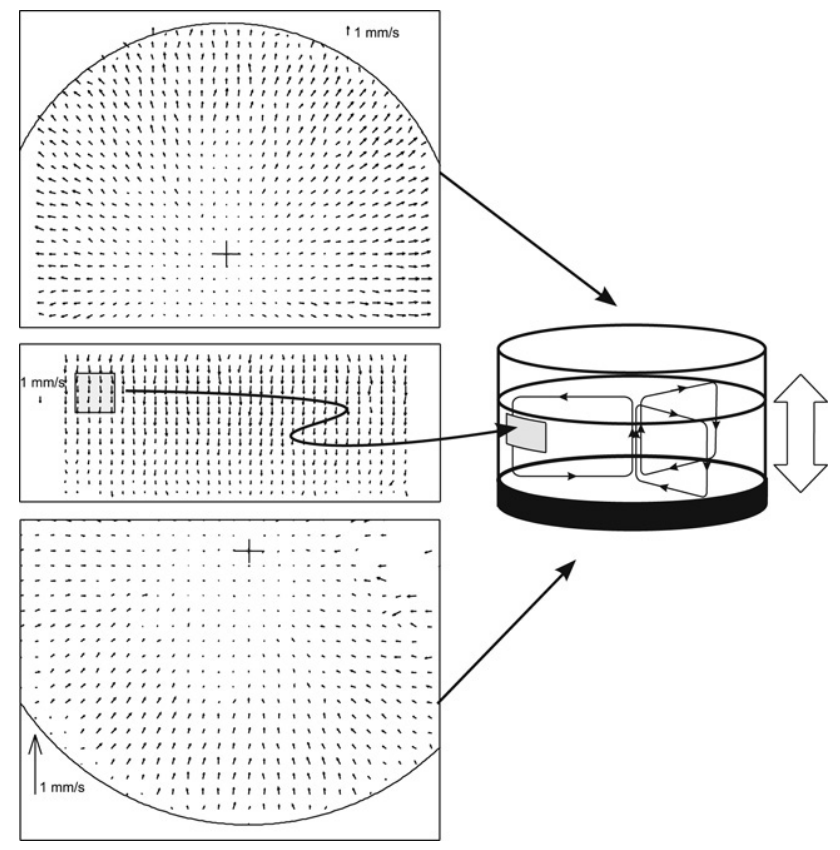

Fig. 5. The convective velocity field at the top of the layer, near the lateral wall and at the bottom of the layer (from top to bottom; only part of the layer is represented.) Note the different scales. This figure corresponds to $N=33$ and $\Gamma=1.90$. The velocity field has been obtained by particle tracking at a small sampling rate ( 25 frames per second) effectively filtering out the rapid movement at the excitation frequency. The small crosses in the top and bottom plots mark the centre of the container and the grey zone shows the window where the particle tracking shown in Fig. 6 has been performed.

Below $\Gamma \simeq 6$, we have observed that the convective motion takes the form of a toroidal roll: the beads go down near the wall and they rise near the center of the container (see Fig. 5). We have tracked the position of individual beads near the lateral wall, at the top surface and at the bottom. The paths of the beads near the lateral wall do not divert much from the vertical; azimuthal velocities are typically less than $10 \%$ of the vertical velocities. Note, however, that near the lateral wall there can also be motion in the radial direction; this component is not accessible in our experiment. This radial movement is evident at the surface and at the bottom.

Let us consider a particle close to the lateral wall, near to the top surface. The vertical position of such a bead at $\Gamma$ below and above the period doubling point is plotted on Fig. 6. The beads roughly follows the same sort of movement described by the IBBM, with a conspicuous difference: there is a distinct drift downwards. We can consider the motion as consisting of two components: a fast one (the jumps at frequency $f$ ) and a slow one, i.e. this drift. The velocity of the latter (the convective motion) is about an order of magnitude smaller than the peak value of the former.

\section{Friction against the walls}

We are now able to discuss the origin of the downward movements near the lateral wall. The motion of a single particle has been shown to consist of a "fast" component (the jumps at the driving frequency $f$ or $f / 2$ ) and a "slow" component (the drift giving rise to convection). Clearly, there must be some mechanism imposing a net shear on the grains in order to induce the flow depicted in Fig. 5. With the aim of investigating this subject, we took a closer look at the trajectories of the particles during each cycle.

A large number of trajectories of single grain flights recorded during one cycle, such as those displayed in Fig. 6, is shown in Fig. 7(a). The common origin has been chosen as the moment when the particles reverse its velocity. Obviously, the measured positions of individual beads are too noisy to obtain clean paths. This is mainly due to the rearrangements of grains during the flight and to the fact that beads rotate. In order to regroup all the trajectories, the origin for all the paths has been arbitrarily chosen at the maximum of the flight. Near this point, almost all of the trajectories should follow the same dynamics. As these maxima are easy to spot, a simple algorithm allows us to displace all the trajectories so that they share this common point. Using this method, the temporal coordinate where the particle touches the base has a lower dispersion. We use the
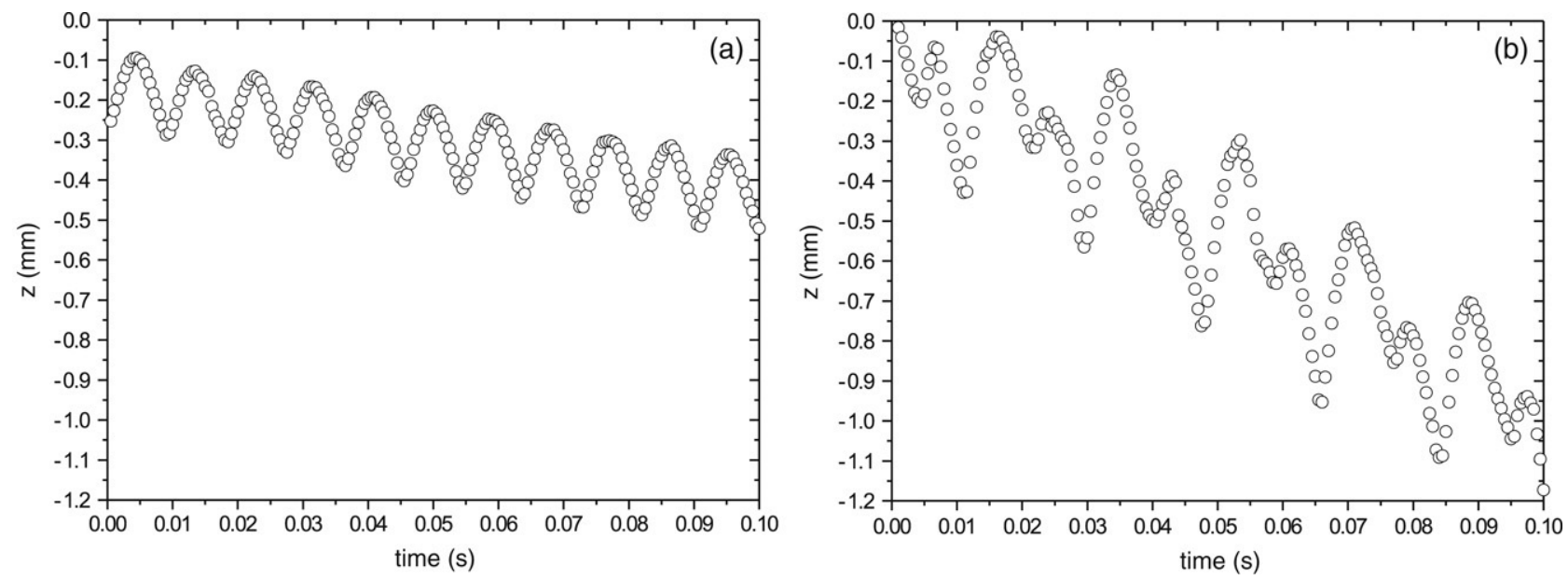

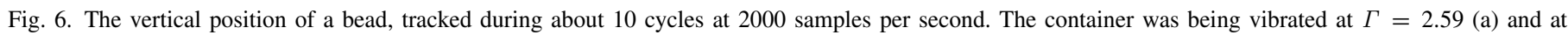
$\Gamma=5.81$ (b). The height of the layer was $N=100$ in both cases. The origin of distances is arbitrary. Note the increasing in the drift velocity when $\Gamma$ grows. 

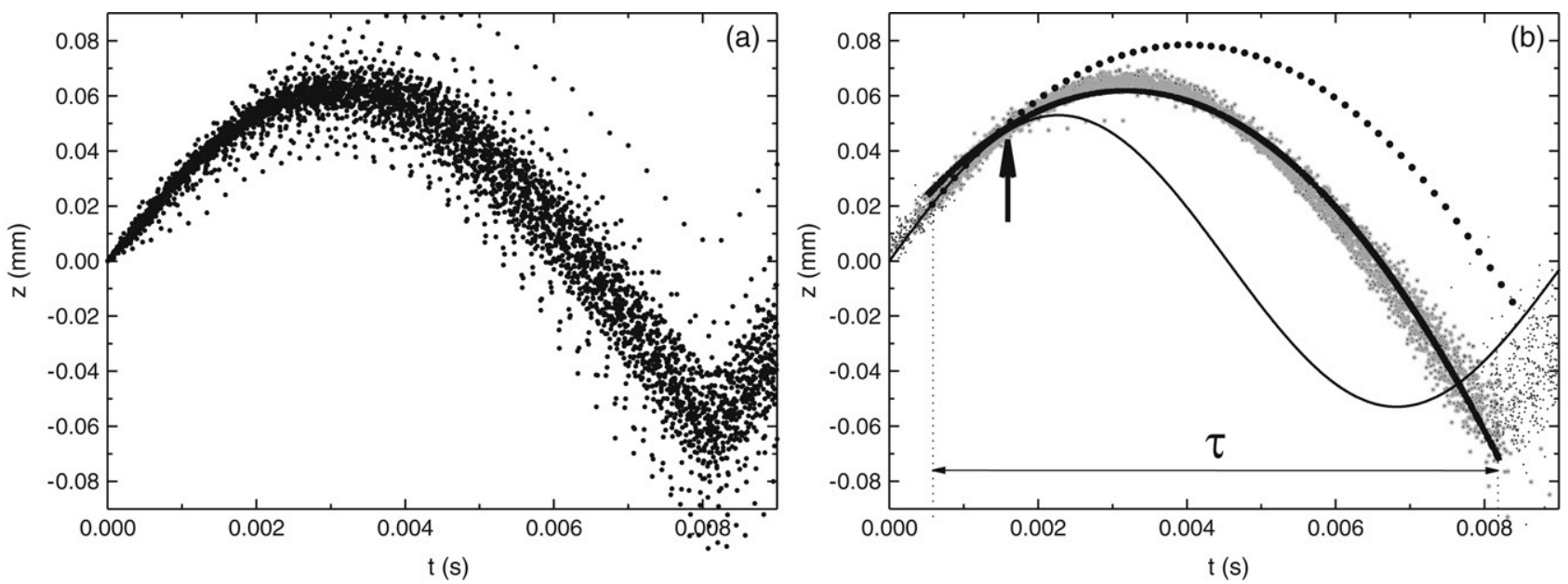

Fig. 7. (a) The trajectories of many grain flights are plotted together. The origins of the positions are the places where velocity changes sign. The data corresponds to $\Gamma=2.5$, a value of the forcing where flight times for an evacuated container and a container with air are the same. It is difficult to fit these data because of their dispersion. (b) The same data, but now the maximum height has been chosen as the common reference. In order to relate them to the movement of the vibrating plate, the phase at the moment of collision has been adjusted to the experimental data. The experimental points have been displaced so their trajectories are tangent to the base at the beginning of the flight. The dotted line corresponds to the flight as predicted by the IBBM. Particles do reach a lower height, and they finally go down further than expected from a "free" flight. The solid line is a quadratic fit. Although the fit could be deemed good, the initial phase for the flight as obtained from it (arrow) does not coincide with $\Gamma=g$.

mean value of these coordinates and the corresponding time determined from the oscilloscope to adjust the phase between the vibrating plate and the flights of the grains.

In Fig. 7(b) we show the regrouped trajectories compared with the trajectory predicted by the IBBM. (We have displaced vertically the measured trajectories so that they are tangent to the plate oscillation.) It seems clear that although the particles begin the flight for a phase almost equal to the one predicted by the IBBM, they finish the flight well below the position were they would land after a free flight. This difference, averaged for an oscillation period, is just the velocity of the slow drift giving rise to the convective flow [18].

From the comparison between the experimental data and the trajectory predicted by the IBBM it is clear that a net acceleration larger than gravity is acting on the particles (as can be seen in Fig. 7(b), the heights reached in the flights, with the same initial condition, are rather different in the measured data than in the IBBM). If this acceleration were constant during the flight time, the path of the particles in a space-time diagram should fit to a parabolic trajectory. We have fitted a parabola, leaving all the parameters free except the maximum, which we have taken as a common origin for all the grains. This fit is shown in Fig. 7(b). The fit seems acceptable to the eye, the effective acceleration averaged along the flight being $g_{f}=$ $10.63 \pm 0.04 \mathrm{~m} / \mathrm{s}^{2}$. This value matches the $g_{\text {eff }}$ estimated from the flight times of the whole granular layer. Nevertheless, the match is not completely satisfying. As stated above, the position of the maximum is fixed and then a constant acceleration and the initial velocity are free parameters of the fit. Under this premises the value obtained for the initial velocity does not agree with the one predicted by the IBBM, and differs from the one that can be extracted from the data.

In order to improve the quality of the fitting, we have tried another approach by introducing a dissipative term which depends on the velocity of the grains. Then the ballistic trajectory of the grain will be modeled by the expression $\ddot{z}+\gamma \dot{z}=-g_{v}$ where $z$ is the position of the particle in the laboratory framework and $\gamma$ is an heuristic coefficient that takes into account the variations of the effective force applied to the grains during the flight. The fit is shown in Fig. 8(a). The value obtained for the initial velocity is in close agreement with the experimental data. The value for $g_{v}$ obtained from the fit, $g_{v}=12.77 \pm 0.07 \mathrm{~m} / \mathrm{s}^{2}$, is considerably larger than that obtained from the flight times of the whole granular layer.

The introduction of a dissipation that depends on the velocity may be deemed a naive approach, but it clearly improves the quality of the fit of the experimental points. The mean acceleration $g_{v}$ found for the particles near the wall, where the trajectories of the grains have been tracked, is larger than the one inferred from the flight times for the whole granular layer, $g_{\text {eff. }}$. Therefore, the difference between $g_{\text {eff }}$ and $g_{v}$ must be due to the wall-particle interaction. This difference between both values would amount to the net stress that the walls cause on the grains close to them, thus imposing the downward flow near the wall. Such an effect can be seen in Fig. 8(b), where the positions of many such grains relative to the center of masses of the granular layer are shown. It is clear from the figure that the effect of the wall on the particles is to delay its flight, specially when the wall and the granular layer separate and move independently. We are developing a more detailed model for particle-wall interaction in order to take into account these effects.

\section{Conclusions and discussion}

For moderate accelerations $(\Gamma \lesssim 2.7)$ the flight of a perfectly inelastic body reproduces quite well the jumps of the granular medium as a whole, just as had been previously reported for the dilation of the upper layer [17]. Nevertheless, flight times are a little bit shorter than predicted. We have verified that the cause 

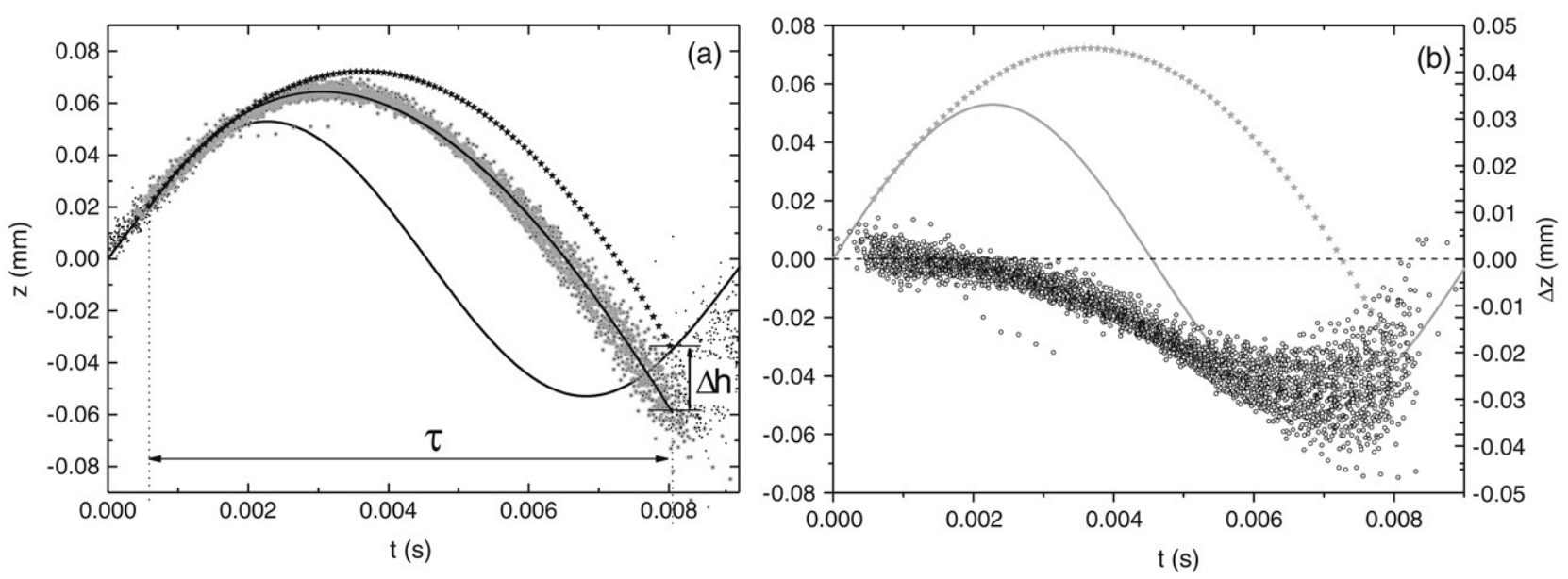

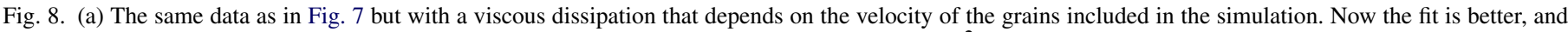

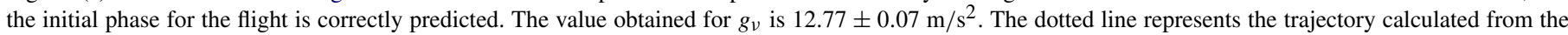

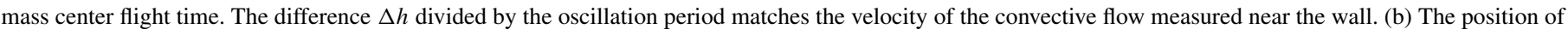

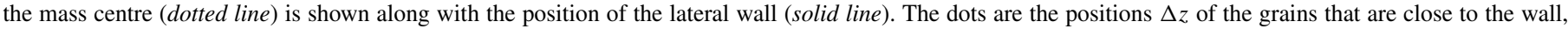

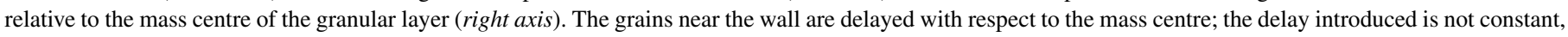
and changes during the flight.

for this is the interstitial air. When the container is evacuated, the agreement between the modified model and the measured data is excellent. We have therefore introduced in the model the interaction between air and the grains so that the agreement when there is air in the container is improved.

Above $\Gamma \approx 2.7$ the IBBM is unable to faithfully reproduce the dynamics observed in the experiment. According to this model, the flight time should increase monotonically until a saddle-node bifurcation appears; then it should remain constant for a finite range of the control parameter. Afterwards it should undergo a period-doubling bifurcation. However, the saddlenode bifurcation has not been observed in our experiments. The finite duration of the collision between the granular layer and the vibrating plate could be the cause for this discrepancy. A deeper study of this subject will be presented elsewhere. Besides, flight times in this parameter region are much lower than predicted both in the presence of air and in vacuum.

The critical value for the control parameter where the period doubling bifurcation takes place is noticeably influenced by the presence of interstitial gas. For a moderate vacuum, such as the one reached in our experiment, the critical value of $\Gamma$ approaches the predicted one for an inelastic model but is still larger than it. Remarkably, flight times in vacuum below the bifurcation are almost indistinguishable from those measured in air. This suggests that the presence of air affects mainly the stability of the solutions for the flight times after the bifurcation.

Just above the period doubling bifurcation, the model fits quite well the shape of the branches near the bifurcation point if the container is evacuated (in air, the flight times are lower than predicted by the IBBM). In neither case, however, the divergence predicted by the model as $\Gamma$ grows larger is observed: flight times change slowly beyond a certain acceleration instead of the expected growth. This behaviour is probably caused by the finite duration of the collision as well.

In this work we have focused on the region below the bifurcation point, where flight times in air and in vacuum are almost the same. The whole granular layer describes a motion with an effective acceleration larger than gravity in average. From the study of the trajectories described by the particles near the lateral wall we have checked that they are subjected to a mean force even larger. We have introduced a fit - that includes a dissipative term - which reproduces quite well the trajectories of the grains. Remarkably, the value for the mean acceleration during the flight is bigger than that obtained from the flight time measurements for the whole granular layer. The difference between both effective accelerations suggests that there is an extra force acting on the grains near the walls, which is larger than the average over all the granular layer. This would be the cause of the downward movement that sets the sense of the convective flow.

\section{Acknowledgments}

This work has been funded by Spanish Government project BFM2002-00414 and FIS2005-03881 as well as Acción Integrada HF2002-0015, by the local Government of Navarre, and by the Universidad de Navarra (PIUNA). I.Z. and J.M.P. thank the Asociación de Amigos de la Universidad de Navarra for a fellowship. The authors wish to thank H. Kellay for his hospitality and his comments and R. Arévalo for his useful comments about the numerical simulation.

\section{References}

[1] M. Faraday, Philos. Trans. R. Soc. London 52 (1831) 299

[2] P. Evesque, J. Rajchenbach, Phys. Rev. Lett. 62 (1989) 44

[3] C. Laroche, S. Douady, S. Fauve, J. Physique France 50 (1989) 699.

[4] E. Clément, J. Duran, J. Rajchenbach, Phys. Rev. Lett. 69 (1992) 1189.

[5] J. Duran, T. Mazozi, E. Clément, J. Rajchenbach, Phys. Rev. E. 50 (1994) 3092.

[6] E. Ehrichs, H. Jaeger, G. Karczmar, J. Knight, V. Kuperman, S.R. Nagel, Science 267 (1995) 1632;

B. Knight, E.E. Ehrichs, V.Yu. Kuperman, J.K. Flint, H.M. Jaeger, S.R. Nagel, Phis. Rev. E 54 (1996) 5726.

[7] L.P. Kadanoff, Rev. Modern Phys. 71 (1999) 435. 
[8] M. Bourzutschky, J. Miller, Phys. Rev. Lett. 74 (1995) 2216.

[9] H. Hayakawa, S. Yue, D.C. Hong, Phys. Rev. Lett. 75 (1995) 2328.

[10] R. Ramírez, D. Risso, P. Cordero, Phys. Rev. Lett. 85 (2000) 1230.

[11] S. Douady, S. Fauve, C. Laroche, Europhys. Lett. 8 (1989) 621.

[12] P.J. Holmes, J. Sound Vibration 84 (1982) 173.

[13] P. Pierański, J. Phys. (Paris) 44 (1983) 573.

[14] N.B. Tufillaro, T.M. Melo, Y.M. Choi, A.M. Albano, J. Phys. (Paris) 47 (1986) 1477.

[15] A. Metha, J.M. Luck, Phys. Rev. Lett. 65 (1990) 393.

[16] F. Melo, P.B. Umbanhowar, H.L. Swinney, Phys. Rev. Lett 75 (1995) 3838.

[17] E. van Doorn, R.P. Behringer, Europhys. Lett. 40 (1997) 387.
[18] A. Garcimartín, D. Maza, J.L. Ilquimiche, I. Zuriguel, Phys. Rev. E 65 (2002) 031303.

[19] B. Thomas, M.O. Mason, A.M. Squires, Powder Technol. 111 (2000) 34.

[20] R.P. Behringer, E. van Doorn, R.R. Hartley, H.K. Pak, Granul. Matter 4 (2002) 9.

[21] X.Y. He, B. Meerson, G. Doolen, Phys. Rev. E 65 (2002) 030301.

[22] D. Risso, R. Soto, S. Godoy, P. Cordero, Phys. Rev. E 72 (2005) 011305.

[23] W. Kroll, Forsch. auf der Geb. des Ing. 20 (1854) 2.

[24] R.G. Gutman, Trans. Instn. Chem. Eng. 54 (1976) 174

[25] L.I. Reyes, I. Sánchez, G. Gutiérrez, Physica A 358 (2005) 466.

[26] J. Bougie, S.J. Moon, J.B. Swift, H. Swinney, Phys. Rev. E 66 (2002) 051301. 